\title{
Mobile Air Pollution Monitoring Network
}

\author{
W. Hedgecock, P. Völgyesi, A. Ledeczi, \\ $X$. Koutsoukos, and A. Aldroubi \\ \{will.hedgecock; peter.volgyesi; \\ akos.ledeczi; xenofon.koutsoukos; \\ akram.aldroubi\}@vanderbilt.edu
}

Institute for Software Integrated Systems - Vanderbilt University Nashville, TN, USA 37203

\author{
A. Szalay \\ Johns Hopkins University \\ Baltimore, MD, USA 21218 \\ szalay@jhu.edu
}

\author{
A. Terzis \\ Johns Hopkins University \\ Baltimore, MD, USA 21218 \\ terzis@cs.jhu.edu
}

\begin{abstract}
Current methods of estimating air quality involve assigning a single value called the Air Quality Index (AQI) to a large land area for a 24 -hour period based on a very few, sparselylocated sensors. This produces a low-resolution image of the air quality in that region. We have devised a new mobile air quality monitoring network with the ability to provide highresolution realtime pollution data at any location within the coverage area. We have prototyped sensors and proven the feasibility of the approach, and are currently testing a smallscale implementation using irregularly-sampled spatiotemporal measurements from mobile car-mounted sensors coupled with existing static data. This data feeds a web-based application, enabling users to view air quality in specific regions as well as estimate exposure over specified time periods and plan routes based on minimal exposure to a given pollutant.
\end{abstract}

\section{Categories and Subject Descriptors}

H.4 [Information Systems Applications]: MiscellaneousWireless Sensor Networks

\section{General Terms}

Algorithms, Measurement, Design, Experimentation

\section{Keywords}

Mobile sensing, modeling, pollution, wireless sensor network

\section{INTRODUCTION}

Air pollution is one of the most important global factors currently affecting quality of life. The current lack of real-

Permission to make digital or hard copies of all or part of this work for personal or classroom use is granted without fee provided that copies are not made or distributed for profit or commercial advantage and that copies bear this notice and the full citation on the first page. To copy otherwise, to republish, to post on servers or to redistribute to lists, requires prior specific permission and/or a fee.

SAC'10 March 22-26, 2010, Sierre, Switzerland.

Copyright 2010 ACM 978-1-60558-638-0/10/03 ...\$10.00. time indicators prohibits accurate decision making and effective air quality control. In the US, there are only about 2,000 sensing stations country-wide. The AIRNow web site [1], run by several government organizations, publishes this pollution data, providing a low-resolution picture of air quality throughout the country. Since pollution is highly locationdependent, however, there is insufficient data to accurately evaluate air quality within specific neighborhoods or at welldefined precise locations.

In contrast to stationary monitoring methods, a detailed picture based on realtime data from mobile sensors over a populated area offers major benefits to air quality control. This idea has been garnering considerable attention recently [2]-[6]. In 2007, we built five car-mounted air pollution sensor prototypes, which we used to demonstrate the feasibility of our approach [2]. We are currently implementing a prototype system consisting of a number of vehicle-mounted sensor nodes, a high-performance database, advanced data processing algorithms to reconstruct an air pollution model from irregularly sampled spatiotemporal observations, and a set of innovative web-based applications for visualizing the data and providing additional services to the public.

\section{SYSTEM ARCHITECTURE}

The current sensor node prototype supports autonomous data collection, storage, and either offline data retrieval or realtime data streaming. Access to the node is provided via an integrated Bluetooth module or a USB cable. The wired interface is used to provide power to the unit for both online operation and charging the integrated Li-ion battery. The battery life of the device lasts only a few hours, but when mounted on a vehicle, this is not a limiting factor. Furthermore, a 2-axis MEMS accelerometer is used to detect if the system is in motion and can automatically turn off powerhungry components. Location and time information is provided by an on-board 20 channel SiRF-III-based GPS module, and gas concentration levels are measured by three analog sensors: $\mathrm{O}_{3}, \mathrm{NO}_{2}$, and $\mathrm{CO} / \mathrm{VOC}$. These readings, along with the temperature, relative humidity, and GPS data are stored in a serial flash device. An Intel 8051-based microcontroller controls all system aspects from battery charging to analog/digital conversions and the USB protocol. 
In addition to input from the mobile sensor nodes, the system also incorporates data from other sources, such as the static sensors deployed by the EPA in metropolitan areas [1]. Other sources of system information include maps, traffic conditions, and weather data from publicly available domains. Sensor data is uploaded to a server whose responsibilities include filtering, calibrating, converting, and storing the data in a scalable fashion. Processes on the server combine data from individual sensors in similar physical regions into virtual sensors while adjusting for GPS inaccuracies using an innovative new GPS smoothing algorithm. Processed and raw data are available via a web interface, allowing users to not only map sensor locations on a Google map, but also to see variable-length histories of each node's measurements, as well as visualize air pollution data in a style similar to a weather radar map.

\section{EXPERIMENTS AND CONSIDERATIONS}

Several experiments have been carried out using our five prototype units to determine the feasibility of our approach including measuring gas concentrations in different environments and under various circumstances. From these experiments, we have been able to verify the expected correlation between measured readings from each of the sensor nodes, as well as the reconstruction of relative gas levels and events at specific times and locations. Additionally, it appears that highly reactive gases such as $\mathrm{O}_{3}$ decrease rapidly when moved away from the pollutant source and are highly subject to environmental conditions, while non-reactive gases like $\mathrm{CO}$ are much more evenly distributed and dispersed around the pollutant source, depending primarily on distance and wind patterns and thus making these gases easier to model.

Although the five prototypes worked quite well during initial testing, significant modifications are needed for scaling. Bluetooth and USB mandate that every vehicle return to one or more stations where data can be downloaded, which is impractical. Moreover, we want the sensor data to stream in realtime. As such, we are replacing the Bluetooth unit with a GSM modem, enabling nodes to transmit data over the mobile telephone networks. In addition, health risks also arise from the presence of particulate matter (PM) in the air which can accumulate in the respiratory system. Unfortunately, since the cheapest PM sensors cost thousands of dollars, we will provide an external interface for pluggable PM sensors, instead of integrating them into our sensor nodes.

Currently, the main research challenge is designing an efficient implementation to transform sparse and irregularly sampled sensor measurements into a 4-dimensional database table to be used for reconstruction of the pollution function. An important aspect of reconstruction is to find an appropriate space-time signal space that describes the functions to be reconstructed. For this purpose, we implemented a set of complex mathematical formulae to find a nonlinear signal model that consists of a union of subspaces instead of a single subspace. One of the advantages of finding a good nonlinear signal model is that the reconstructions will be sparse in terms of representations within the model. This means that the reconstructed functions will be linear combinations of a few basis functions. Thus, storing or transmitting the reconstructed functions will be cheap and efficient. Moreover, the model search will implicitly allow us to take complex factors into account such as terrain and the fact that we are sampling almost exclusively near the main pollutant sources.

\section{CONCLUSION}

The overarching goal of this project is to dramatically increase the resolution of air pollution information and maximize its impact on public life. Our pollution monitoring system operates deeply embedded in the physical environment. Irregular spatiotemporal samples are used to reconstruct an overall pollution model affected by emission sources, weather, terrain, traffic patterns, and other factors. We have designed mobile nodes to sense known air pollutants as well as environmental conditions and communicate this data to a central server, providing continuous realtime data feeds over a web interface. Finally, the system provides an intuitive method of data retrieval using web-based visualization with a number of other novel applications still under development, such as a "Green Trip Planner," which takes into account regional air quality data and calculates trip paths minimizing exposure to pollution. We currently provide two approaches for widespread dissemination of our information. First, the project web site [8] displays sensor and pollution data and allows users to download pollution information from individual or groups of nodes. Secondly, we have received permission from the EPA to publish our pollution data on their AIRNow web site [1], thus providing widespread access to our data as a free service to the public.

\section{ACKNOWLEDGMENT}

This project was made possible by a generous grant from Microsoft, NSF award 0807464, and the continuing financial support of Vanderbilt University.

\section{REFERENCES}

[1] AIRNow. (2009). Quality of air means quality of life. [Online]. Available: http://airnow.gov/

[2] P. Völgyesi, A. Nádas, X. Koutsoukos, and A. Lédeczi. "Air quality monitoring with sensormap." In IPSN, pp. 529-530, 2008.

[3] M. Ghanem, Y. Guo, J. Hassard, M. Osmond, and M. Richards. "Sensor grids for air pollution monitoring." In Proceedings of the 3rd UK e-Science All Hands Meeting, 2004.

[4] P. Rudman, S. North, and M. Chalmers. "Mobile pollution mapping in the city." In Proceedings of the UK-UbiNet workshop on eScience and ubicomp, 2005.

[5] P.M. Aoki, R.J. Honicky, A. Mainwaring, C. Myers, E. Paulos, S. Subramanian, and A. Woodruff. "Common sense: mobile environmental sensing platforms to support community action and citizen science (demonstration)." In Adjunct Proceedings - Ubicomp 2008, Sept. 2008.

[6] (2009). "Personal environmental impact report". UCLA CENS. [Online]. Available: http://peir.cens.ucla.edu/

[7] J. Cayzac. (2006). "The cumulative displacement filter." [Online]. Available: http://julien.cayzac.name/code/gps/

[8] Mobile Air Quality Monitoring Network. (2009). "MAQUMON Gateway." [Online]. Available: http://people.vanderbilt.edu/ ${ }^{\sim}$ will.hedgecock 